\title{
Tumor- und Metastasenchirurgie an der Wirbelsäule
}

\author{
Christoph Josten, Alexander Franck
}

\section{Zusammenfassung}

Patienten mit Tumorerkrankungen entwickeln in $70 \%$ der Fälle eine Tumorabsiedelung in Form von Metastasen. Dabei hat sich gezeigt, dass die Wirbelsäule mit bis zu 40\% der Ort der häufigsten Absiedlung in den Knochen ist. Weitaus seltener sind primäre Wirbelsäulentumoren. Hat sich eine Metastase im Wirbelkörper manifestiert, führt sie im Verlauf durch Veränderung der knöchernen Struktur zu einer zunehmenden Instabilität, die einerseits verantwortlich ist für die Schmerzen der Tumorpatienten und andererseits eine potenzielle Gefährdung des Rückenmarks mit drohendem Querschnitt darstellt. Diese Probleme lassen sich durch konservative Maßnahmen häufig nicht ausreichend behandeln bzw. verhindern. Die operative Stabilisierung mit das Rückenmark entlastender Laminektomie ist deshalb bei schweren, konservativ nicht zu beherrschenden instabilitätsbedingten Schmerzen, zunehmender Einengung des Spinalkanals durch rasches Tumorwachstum oder neurologische Ausfälle die Therapie der Wahl.

\section{Spinal Tumour and Metastases Surgery}

$70 \%$ of all patients with tumours will develop metastases. Up to $40 \%$ of all boney metastases are located in the spinal column, making this the most common location. Primary spinal column tumors are much less common. Metastases in the spinal column can result in changes in the boney structure and to an increasing instability which is not only responsible for the pain but also constitutes an endangerment of the spinal cord and subsequent paralysis. Frequently, these problems cannot be treated or prevented sufficiently by conservative therapy alone. The operative stabilisation of the spinal column with laminectomy is the procedure of choice to reduce severe pain due to instability that cannot be managed conservatively, to counter increasing restriction of the spinal cord by rapid tumour growth or to avoid neurological deficits.

Die Metastasen in der Wirbelsäule stammen meist von den Krebsarten Brust-, Niere-, Prostata- und Lungenkrebs. Insgesamt sind Männer häufiger betroffen als Frauen (6:4), wobei der Altersgipfel zwischen dem 40. und 65. Lebensjahr liegt. Betroffen ist am häufigsten die Brustwirbelsäule, gefolgt von der Lendenwirbelsäule und am seltensten die Halswirbelsäule (Abb.1). Dabei werden die Absiedlungen häufig in verschiedenen Etagen der Wirbelsäule gefunden.

Haben sich Metastasen im Wirbelkörper manifestiert, führen sie im Verlauf durch Veränderung der knöchernen Struktur zu einer zunehmenden Instabilität. Diese ist einerseits verantwortlich für die Schmerzen der Tumorpatienten, welche nahezu immer als erstes Tumorzeichen beschrieben werden. Andererseits stellen sie eine potenzielle Gefährdung des Rückenmarks mit drohendem Querschnitt dar. Neurologische Funktionsausfälle können sich dann je nach Stadium durch sensible Ausfälle, Paraparesen, Paraplegien oder Blasen-/Mastdarmstörungen äußern.

Die beschriebenen Symptome und Funktionsausfälle lassen sich durch konservative Maßnahmen nicht ausreichend behandeln bzw. verhindern. Daher ist die operative Stabilisierung mit das Rückenmark entlastender Laminektomie bei schweren, konservativ nicht zu beherrschenden instabilitätsbedingten Schmerzen, zunehmender Einengung des Spinalkanals durch rasches Tumorwachstum oder neurologische Ausfälle die Therapie der Wahl.

\section{Hauptteil}

In der Regel werden die betroffenen Patienten aufgrund von Rückenschmerzen in der Klinik vorstellig. Dabei ist nicht immer ein primäres Tumorgeschehen bekannt. Gerade im Vorfeld ist daher eine detaillierte Diagnostik unverzichtbar. Zuallererst ist die genaue Befragung und Untersuchung essenziell. Schon hier lassen sich wichtige Hinweise für eine zugrunde liegende Krebserkrankung erarbeiten.

Ist diese bereits diagnostiziert, so ist es von entscheidender Bedeutung, bei unklaren Rückenschmerzen, meist ohne Trauma, eine Metastasierung im Bereich der Wirbelsäule auszuschließen.

Die diagnostische Vorgehensweise ist standardisiert durchzuführen:

Die konventionelle Röntgenaufnahme kann hinweisend für knöcherne Veränderungen sein, es ist jedoch zu beden- 


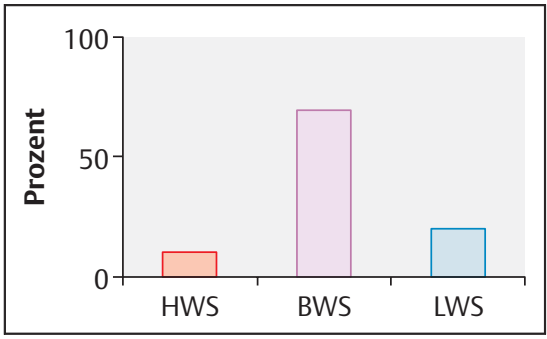

Abb. 1 Häufigkeitsverteilung von Metastasen in der Wirbelsäule.
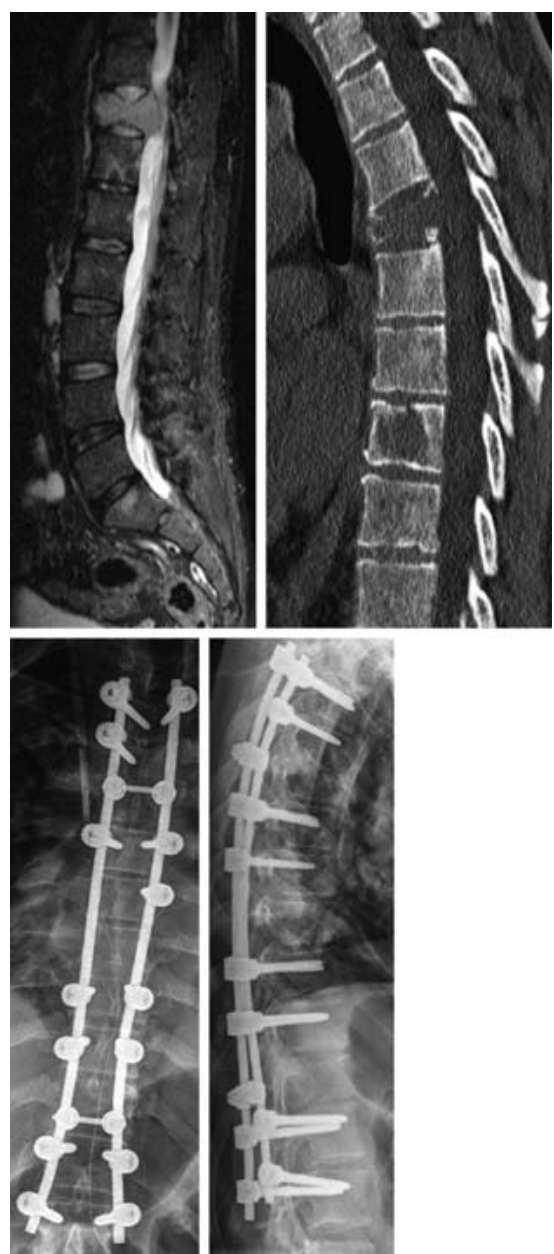

Abb. 2 Multiple Metastasierung BWS und LWS.

ken, dass strukturelle Veränderungen erst bei einem Substanzverlust von 30$50 \%$ nachzuweisen sind, gerade wenn noch kein Wirbelbruch vorliegt.

Weitaus sicherer und vor allem früher können Metastasen an der Wirbelsäule durch die Magnetresonanztomografie nachgewiesen werden.

Dabei ist wichtig, immer die gesamte Wirbelsäule abzubilden, da in bis zu $1 / 5$ der Fälle mehrere Metastasen in unterschiedlichen Etagen gefunden werden.

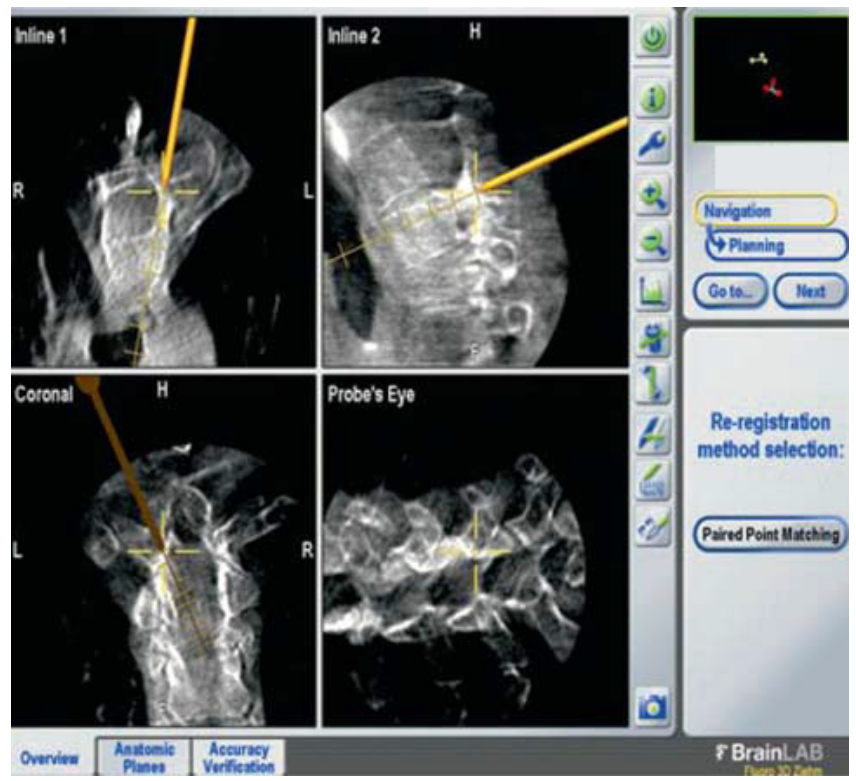

Abb. 3 Navigation im Bereich der Brustwirbelsäule.
Zur Beurteilung der Stabilitätsverhältnisse im Bereich der betroffenen Wirbelsäulenabschnitte ist die Computertomografie die Untersuchung der Wahl. Jedoch muss auch hier ein Knochensubstanzverlust von $15-20 \%$ vorliegen, um zu einer sicheren Metastasendiagnostik zu gelangen. Je nach Stand der vorausgegangenen Diagnostik ist das Tumorscreening durch Skelettszintigrafie, CT von Abdomen/Thorax oder ggf. Positronenemissionstomografie zu vervollständigen.

Betrachtet man den Befall des Wirbelkörpers im Einzelnen, so hat sich gezeigt, dass die knöchernen Anteile in über $2 / 3$ der Fälle betroffen sind und hier vor allem der Übergang zwischen Pedikel und Wirbelkörper. Da die Dura mater bezüglich der Tumorausbreitung ein natürliches Hindernis darstellt, liegen die Metastasen überwiegend extradural.

Die Therapie sollte individuell auf den Patienten abgestimmt sein. Dabei ist es sinnvoll, möglichst mehrere Disziplinen einzubeziehen, um den Krankheitsverlauf günstig $\mathrm{zu}$ beeinflussen. Folgende Punkte sind unserer Meinung nach eine Indikation zur operativen Vorgehensweise:

- durch konservative Maßnahmen nicht $\mathrm{zu}$ beherrschende Schmerzen, die meist zu einer vollständigen Immobilisierung der Betroffenen führen,

- ein drohender Bruch mit Gefahr der Verlegung des Rückenmarks,

- vorhandene Wirbelkörperbrüche mit Instabilität und Deformität des betroffenen Wirbelsäulenabschnitts,
- eine fortschreitende Einengung des Spinalkanals mit neurologischen Ausfällen.

Das Ziel der Operation muss vorrangig die Stabilität und die Entlastung des Rückenmarks sein. Dabei ist die Wahl der entsprechenden Vorgehensweise von verschiedenen Gesichtspunkten abhängig und dahingehend zu planen:

- Allgemeinzustand des Patienten und Tumorprogredienz,

- Lokalisation der Metastase:

- Wirbelsäulenabschnitt (HWS/BWS/ LWS),

- im Wirbelkörper (Wirbelkörper, Pedikel, Laminae),

- Ausdehnung in den Spinalkanal, - ggf. erforderliche Rekonstruktion.

Nur sehr selten handelt es sich um eine einzelne Wirbelkörpermetastase, die komplett entfernt werden kann. Hierbei ist ein primär kurativ zu behandelnder Tumor mit prognostisch günstiger Tumorbiologie und fehlender systemischer Ausbreitung mit zu erwartendem kurzem Tumorverlauf Voraussetzung. Zusätzlich müssen weitere Metastasen durch eine entsprechende Diagnostik ausgeschlossen sein und ein längeres metastasenfreies Intervall zwischen Primärtumor und letztmaliger Bildgebung vorliegen.

Regelhaft weist die Wirbelsäule mehrere betroffene Segmente auf, wobei die instabilsten Abschnitte über mehrere Höhen von dorsal mit einem Fixateur interne stabilisiert werden, ohne die eigentliche Metastase zu adressieren. 
Zusätzlich erfolgt eine Entlastung des Rückenmarks durch eine Laminektomie, auch wenn dieses noch nicht direkt betroffen ist, um das Auftreten von neurologischen Ausfällen zu verhindern (Abb. 2).

Gerade im Bereich der unteren Hals- und oberen Brustwirbelsäule führen wir die Stabilisierung mithilfe der Navigation durch (Abb. 3).

$\mathrm{Zu}$ berücksichtigen ist die Möglichkeit der präoperativen Embolisation stark durchbluteter Tumormetastasen (z.B. Nierenzellkarzinom), um einen massiven intraoperativen Blutverlust zu vermeiden.

\section{Schlussfolgerung}

Die postoperative Nachsorge unserer Patienten hat gezeigt, dass in über $80 \%$ der Fälle eine deutliche Schmerzreduktion erzielt werden konnte. Dies ermöglicht eine frühzeitige Mobilisierung mit rascher Entlassung aus der stationären in die ambulante Weiterbehandlung im familiären Umfeld. Alle unsere Patienten, die zum Operationszeitpunkt keine neurologischen Ausfälle aufwiesen und einer das Rückenmark entlastenden prophylaktischen Laminektomie unterzo- gen wurden, boten auch im postoperativen Follow-up keine neurologischen Befunde.

Weit fortgeschrittene neurologische Defizite können jedoch häufig durch die Operation nicht gebessert werden. Gerade im Bereich der Brustwirbelsäule besteht sogar die Gefahr der Verschlechterung durch das Operationstrauma. Von Bedeutung ist deshalb, Wirbelsäulenmetastasen frühzeitig zu erkennen und entsprechend zu behandeln, um neurologische Ausfälle zu vermeiden.

Dabei ist das Tumorboard als interdisziplinäres Gremium gerade unter palliativen Gesichtspunkten essenziell zur notwendigen frühzeitigen Koordinierung der verschiedenen Therapieansätze.

\section{Literatur}

${ }^{1}$ Aebi M. Spinal metastases in the elderly. Eur Spine J 2003; 12: 202-213

${ }^{2}$ Ewerbeck V, Friedl W. Chirurgische Therapie von Skelettmetastasen. Berlin, Heidelberg, New York: Springer; 1992

${ }^{3}$ Finkelstein JA, Zaveri G, Wai E et al. A population-based study of surgery for spinal metastases. Survival rates and complications. J Bone Joint Surg [Br] 2003; 85: 1045-1050

4 Halm H, Richter A, Lerner Tet al. En-bloc-Spondylektomie und Defektrekonstruktion bei Primärtumoren und Solitärmetastasen der Wirbelsäule. Orthopäde 2008; 37: 356-366
${ }^{5}$ Hessler $C$ et al. Komplikationen bei operativer Dekompression an der tumorinfiltrierten Wirbelsäule. Chirurg 2007; 78: 915-927

${ }^{6}$ Mutschler W, Wörsdörfer O, Schulte M. Tumorresektionen und rekonstruktive Eingriffe be Wirbelsäulentumoren. Orthopäde 1993; 22: 189-199

${ }^{7}$ Pfannenberg AC, Eschmann SM, Claussen CD et al. Bildgebende Diagnostik von Metastasen in Hirn, Knochen, Leber und Lunge. Onkologe 2004; 10: 504-516

8 Rehak S et al. The role of embolization in radical surgery of renal cell carcinoma spinal metastases. Acta Neurochir 2008; 150: 11771181

${ }^{9}$ Rybak LD, Rosenthal DI. Radiological imaging for the diagnosis of bone metastases. QJ Nucl Med 2001; 45: 53-64

${ }^{10}$ Schaser K-D et al. Chirurgisches Management von Wirbelsäulenmetastasen. Unfallchirurg 2007; 110: 137-162

Prof. Dr. med. Christoph Josten Direktor der Chirurgischen Klinik I Dr. med. Alexander Franck Assistenzarzt

Klinik und Poliklinik für Unfall-, Wiederherstellungs- und Plastische Chirurgie

Universitätsklinikum Leipzig AöR

Liebigstr. 20

04103 Leipzig

josten@medizin.uni-leipzig.de 